\title{
Spin-Wave Spectrum of the Quantum Ferromagnet on the Pyrochlore Lattice $\mathbf{L} \mathbf{u}_{2} \mathbf{V}_{2} \mathbf{O}_{7}$
}

\author{
M. Mena, ${ }^{1,2}$ R. S. Perry, ${ }^{1,3}$ T. G. Perring, ${ }^{4,1}$ M. D. Le, ${ }^{5}$ S. Guerrero, ${ }^{6}$ M. Storni, ${ }^{6}$ D. T. Adroja,${ }^{4}$ \\ Ch. Rüegg, ${ }^{2,7}$ and D. F. McMorrow ${ }^{1}$ \\ ${ }^{1}$ London Centre for Nanotechnology and Department of Physics and Astronomy, University College London, \\ Gower Street, London WCIE 6BT, United Kingdom \\ ${ }^{2}$ Laboratory for Neutron Scattering and Imaging, Paul Scherrer Institute, CH-5232 Villigen PSI, Switzerland \\ ${ }^{3}$ Centre for Science at Extreme Conditions, University of Edinburgh, Mayfield Road, Edinburgh EH9 3JZ, Scotland \\ ${ }^{4}$ ISIS Facility, STFC Rutherford Appleton Laboratory, Harwell Oxford, Didcot OX11 OQX, United Kingdom \\ ${ }^{5}$ Helmholtz-Zentrum Berlin, Hahn-Meitner-Platz 1, D-14109 Berlin, Germany \\ ${ }^{6}$ Condensed Matter Theory, Paul Scherrer Institute, CH-5232 Villigen, Switzerland \\ ${ }^{7}$ DPMC-MaNEP, University of Geneva, CH-1211 Geneva 4, Switzerland
}

(Received 14 December 2013; published 23 July 2014)

\begin{abstract}
Neutron inelastic scattering has been used to probe the spin dynamics of the quantum $(S=1 / 2)$ ferromagnet on the pyrochlore lattice $\mathrm{Lu}_{2} \mathrm{~V}_{2} \mathrm{O}_{7}$. Well-defined spin waves are observed at all energies and wave vectors, allowing us to determine the parameters of the Hamiltonian of the system. The data are found to be in excellent overall agreement with a minimal model that includes a nearest-neighbor Heisenberg exchange $J=8.22(2) \mathrm{meV}$ and a Dzyaloshinskii-Moriya interaction (DMI) $D=1.5(1) \mathrm{meV}$. The large DMI term revealed by our study is broadly consistent with the model originally used to explain the magnon Hall effect in this compound [Onose et al., Science 329, 297 (2010) and Ideue et al., Phys. Rev. B 85, 134411 (2012)]. However, our ratio of $D / J=0.18(1)$ is roughly half of their value, and is much larger than those found in other theoretical studies [Xiang et al., Phys. Rev. B 83, 174402 (2011) and Mook et al., Phys. Rev. B 89,134409 (2014)].
\end{abstract}

DOI: 10.1103/PhysRevLett.113.047202

PACS numbers: 75.10.Dg, 75.30.Ds

Depending on the nature of the interactions, the pyrochlore lattice can be highly frustrated, leading to a rich diversity of fascinating properties when the lattice sites are decorated with "classical" (large $S$ ) spins [1]. Arguably the most celebrated example is the discovery of spin-ice behavior due to ferromagnetically coupled Ising spins [2,3] and the emergence of magnetic monopoles [4,5]. While many examples of classical pyrochlores exist, there are few examples of pyrochlores where the spins of the magnetic ions are explicitly in the quantum $(S=1 / 2)$ limit. Quantum effects can, however, play a decisive role even in classical pyrochlores if their low-energy physics maps onto an effective spin $1 / 2$ model [1]. In either case, quantum effects may produce a range of novel phenomena including the realization of a three-dimensional quantum spin-liquid ground state, emergent electromagnetism supporting photonlike excitations, etc. [6-9]. Interest in itinerant pyrochlore magnets is also motivated by the various anomalous transport properties they exhibit [10].

$\mathrm{Lu}_{2} \mathrm{~V}_{2} \mathrm{O}_{7}$ is a ferromagnetic, small-gap Mott insulator that crystallizes in the pyrochlore structure and displays a number of exceptional properties. Figure 1(a) shows the $\mathrm{V}^{4+}(S=1 / 2)$ sites in the pyrochlore lattice, which form a three-dimensionally coordinated network of corner sharing tetrahedra. Bulk measurements have established that the spins order ferromagnetically at $T_{C}=70 \mathrm{~K}[11,12]$. Measurements of the thermal conductivity in $\mathrm{Lu}_{2} \mathrm{~V}_{2} \mathrm{O}_{7}$ by Onose et al. have been interpreted in terms of a magnon
Hall effect [11], based on the observation that the thermal conductivity has a distinctive dependence on applied magnetic fields for temperatures below $T_{C}$. This highly unusual and previously unreported phenomenon was shown to be consistent with a model in which the Dzyaloshinskii-Moriya interaction (DMI) between nearest neighbor spins deflects magnon wave packets propagating from the hot to the cold side of the sample $[11,13]$.

Further evidence of the exceptional properties of $\mathrm{Lu}_{2} \mathrm{~V}_{2} \mathrm{O}_{7}$ was provided by Zhou et al. [12] who discovered that it displays a large (50\%) magnetoresistance around $70 \mathrm{~K}$ in an applied magnetic field. Additionally, polarized neutron diffraction found evidence for an orbital ordered ground state with associated superexchange pathways favoring a dominant nearest-neighbor ferromagnetic coupling [14]. It has also been suggested that $\mathrm{Lu}_{2} \mathrm{~V}_{2} \mathrm{O}_{7}$ may be an example of a topological magnon insulator with chiral edge states [15].

The magnon Hall effect data in $\mathrm{Lu}_{2} \mathrm{~V}_{2} \mathrm{O}_{7}$ has been interpreted in terms of a Hamiltonian that takes the form

$$
\mathcal{H}=\sum_{\langle i j\rangle}\left(-J \vec{S}_{i} \cdot \vec{S}_{j}+\vec{D}_{i j} \cdot\left(\vec{S}_{i} \times \vec{S}_{j}\right)\right)+g \mu_{B} \sum_{i} \vec{H} \cdot \vec{S}_{i}
$$

where $J$ is the nearest-neighbor Heisenberg exchange, $\vec{D}_{i j}$ represents the DMI, whose directions are sketched in Fig. 1(b), $\vec{S}_{i}$ are spin operators, $\vec{H}$ is the magnetic field, 


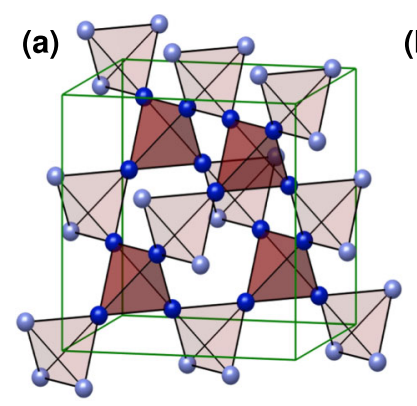

(b)

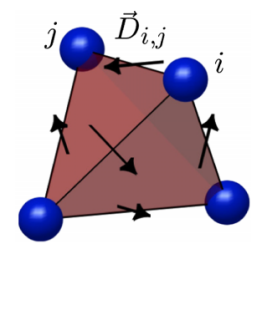

(c)

(d)

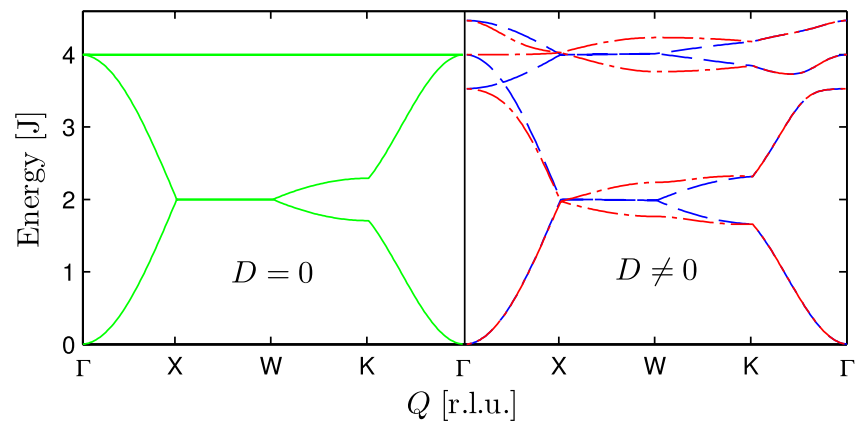

FIG. 1 (color online). (a) Pyrochlore structure of $\mathrm{Lu}_{2} \mathrm{~V}_{2} \mathrm{O}_{7}$. The $\mathrm{V}^{4+}$ ions (blue) form a network of vertex-sharing tetrahedra with four tetrahedra in the unit cell. (b) A single tetrahedron. The arrows located between adjacent $\mathrm{V}^{4+}$ sites denote the directions of the DMI vectors; e.g., the vector $\vec{D}_{i, j}$ is associated with the interaction between sites $i$ and $j$. Calculated spin-wave dispersion for $\mathrm{Lu}_{2} \mathrm{~V}_{2} \mathrm{O}_{7}$ for (c) $D=0$ and (d) $D=J / 3$ with the local magnetic field $\vec{H}$ parallel to either (100) (blue) or (010) (red). $\Gamma$, $X, W$, and $K$ label the high-symmetry points of the conventional fcc Brillouin zone.

and $\langle i j\rangle$ runs over all pairs of nearest neighbors [11]. To date there have been no reports of experiments designed to determine accurately the parameters of the Hamiltonian. Following standard procedures, this Hamiltonian can be diagonalized by expressing the spin operators in the local coordinates of each of the four ferromagnetic sublattices and applying the Holstein-Primakoff transformation. Figures 1(c)-1(d) show the calculated dispersion for the four branches for different values of $D=\left|\vec{D}_{i j}\right|$ and $\vec{H}$.

The magnitude of the anomalous contribution to the thermal conductivity due to magnons is determined by the ratio $D / J$, which was estimated in $\mathrm{Lu}_{2} \mathrm{~V}_{2} \mathrm{O}_{7}$ to be $D / J \simeq$ $1 / 3$ by fitting the transport data $[11,13]$ and, hence, would require strong spin-orbit coupling. In contrast, densityfunctional theory (DFT) calculations obtained a much lower ratio $D / J \simeq 1 / 20$, with the authors instead emphasizing the importance of single ion anisotropy [16]. More recently, a theoretical study [17] refitted the data by Onose et al. using a different theory and found $D / J$ to be in the range $0.0038-0.0056$. There is thus a prima facie case for accurately determining the Hamiltonian of $\mathrm{Lu}_{2} \mathrm{~V}_{2} \mathrm{O}_{7}$ both in terms of interpreting the transport data, and more generally of understanding the spin dynamics of this elusive example of a pyrochlore lattice in the quantum limit. Here we present the results of a neutron inelastic scattering experiment that satisfies these objectives.

$\mathrm{Lu}_{2} \mathrm{~V}_{2} \mathrm{O}_{7}$ crystallizes in the cubic $F d \overline{3} m$ space group (number 227), with a lattice parameter of $9.94 \AA$ A. Single crystals were grown in an image furnace, and were characterized by SQUID magnetometry and x-ray diffraction, confirming the ferromagnetic transition temperature and good crystalline quality. Two single crystals, total mass of $3.6 \mathrm{~g}$, were co-aligned with the $(H H L)$ plane horizontal. The experiment was performed on the MERLIN direct geometry, time-of-flight spectrometer at the ISIS facility (UK) [18]. Data were collected for incoming energies $E_{i}$ (measured elastic resolutions, FWHM) of 25 (3.0), 50 (5.3), and 80 (7.2) $\mathrm{meV}$ and at a temperature of $4 \mathrm{~K}$. The small moment size and mass of the sample necessitated typical counting times of $15 \mathrm{~h}$ to acquire sufficient statistics for a given energy and angular setting of the sample. Data sets were collected with the incident neutron beam along the $\langle 001\rangle,\langle 110\rangle$, and $\langle 111\rangle$ directions. The data were corrected for detector efficiency, scattered versus incident wave vector ratio $k_{f} / k_{i}$, and normalized to a vanadium standard using the program MANTID [19]. The resulting $\mathrm{S}(\vec{Q}, \mathrm{w})$ data sets were analyzed with the HORACE software package [20].

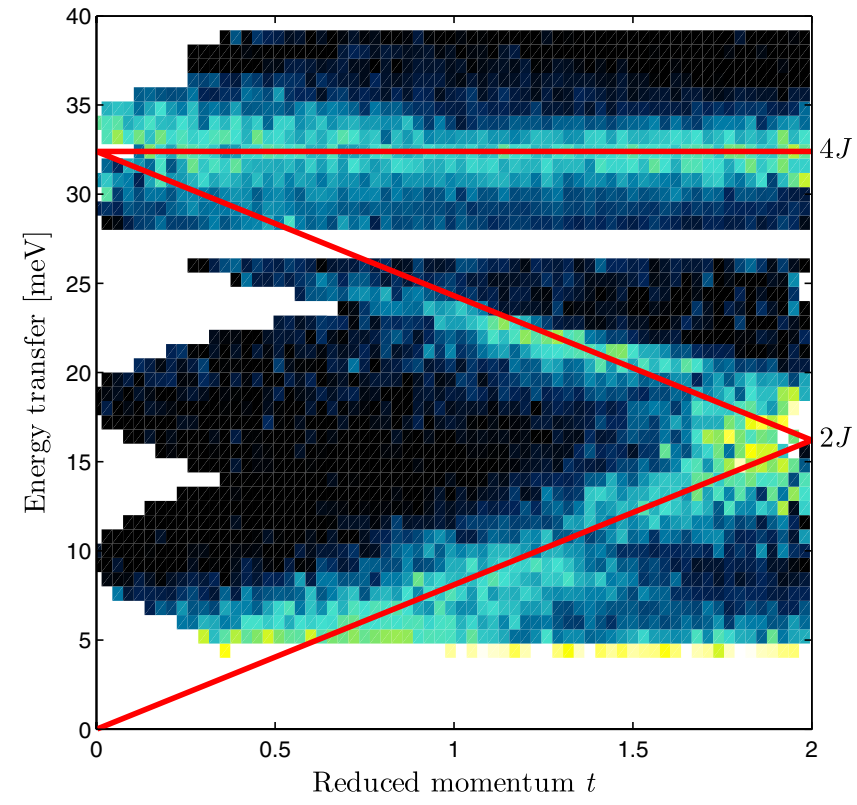

FIG. 2 (color online). Neutron inelastic scattering data $\left(E_{i}=50 \mathrm{meV}\right.$ ) from $\mathrm{Lu}_{2} \mathrm{~V}_{2} \mathrm{O}_{7}$ plotted as a function of the reduced momentum $t$ defined in the text. The data between 0 and 27 meV energy transfer (below the white gap) are obtained by averaging over data with $|\vec{Q}|<3$ r.l.u., while between 28 and $40 \mathrm{meV}$ energy transfer (above the white gap) they are obtained by considering only data with $|\vec{Q}|<5$ r.l.u.. The solid red line represents a fit of the dispersion with $J=8.1(1)$ and $D=0 \mathrm{meV}$. 
In Fig. 2 we show an overview of the data covering the full energy range over which we observed magnetic scattering. For the abscissa we have chosen to use a reduced wave vector coordinate, $t$. This allows us to exploit the symmetry of the system, and to utilize data from more than one Brillouin zone and orientation of the crystal. The reduced coordinate $t$ is defined by $t(\vec{Q})=2-$ $[1+\cos (\pi H) \cos (\pi K)+\cos (\pi K) \cos (\pi L)+\cos (\pi L) \times$ $\cos (\pi H)]^{1 / 2}$, where $t \in[0,2]$. Rebinning the data in terms of $t$ folds several Brillouin zones onto the same axis; for example, any zone center will be rebinned to $t=0$. In order to exclude scattering from aluminium phonons from the sample environment only data with $|Q|<3$ r.l.u. were averaged below $27 \mathrm{meV}$, and $|\vec{Q}|<5$ r.l.u. between 27 and $40 \mathrm{meV}$. For $D=0 \mathrm{meV}$ the dispersions of the four modes associated with the four ferromagnetic sublattices of a tetrahedron become $\hbar \omega_{1}(t)=t J, \quad \hbar \omega_{2}(t)=(4-t) J$, $\hbar \omega_{3,4}(t)=4 J$ [11]. The solid line in Fig. 2 represents a fit of this dispersion to the data from which we obtained the nearest-neighbor exchange coupling $J=8.1(1) \mathrm{meV}$. Thus even at this level of analysis we can assert that the Hamiltonian relevant to $\mathrm{Lu}_{2} \mathrm{~V}_{2} \mathrm{O}_{7}$ is dominated by isotropic nearest-neighbor exchange.

Further data analysis and modeling were performed in natural reciprocal space coordinates $(H, K, L)$. Figure 3 shows a compendium of data [left hand panels, (a), (c), and (e)] plotted as a function of $H$ and $K$ for different intervals of both energy and $L$. For energies lower than approximately $16 \mathrm{meV} \approx 2 J$ [Fig. 3(a)], rings of intensity are evident, centered on (lllll)-type $\Gamma$ points, as expected from the almost-quadratic form of the dispersion at low energies; see Figs. 1(c) and 1(d). At energies $\sim 2 J$ [Fig. 3(c)], the signal has moved to the edges of the Brillouin zones. For energies $\sim 4 J$ [Fig. 3(e)] the dispersion is expected to be almost flat-with maximal deviations $\pm \sqrt{2} D$-and the significant $\vec{Q}$ dependence of the spectral weight gives rise to a square latticelike structure in the intensity. [In Fig. 3(e) a smoothly varying $|\vec{Q}|$-dependent background was subtracted from the data.]

Simulations of the data were performed using the package MCPHASE [21], which uses a mean-field random-phase approximation to calculate the energies and spectral weights of the magnetic excitations, which were then convoluted with a Gaussian of the same width as the calculated instrumental energy resolution at the appropriate energy transfer in order to model $S(\vec{Q}, \omega)$. MCPHASE was configured to simulate $S(\vec{Q}, \omega)$ expected from Eq. (1), with an isotropic spin-only magnetic form factor for the $\mathrm{V}^{4+}$ ions calculated within the dipole approximation. In Figs. 3(b), 3(d), and 3(f) simulations are shown for $J=8.1$ and $D=0 \mathrm{meV}$. Remarkably, this minimal model which neglects the DMI and uses an isotropic form factor provides an excellent description of the data over all relevant wave vectors and energies. It is therefore apparent that to a good approximation the Hamiltonian in $\mathrm{Lu}_{2} \mathrm{~V}_{2} \mathrm{O}_{7}$
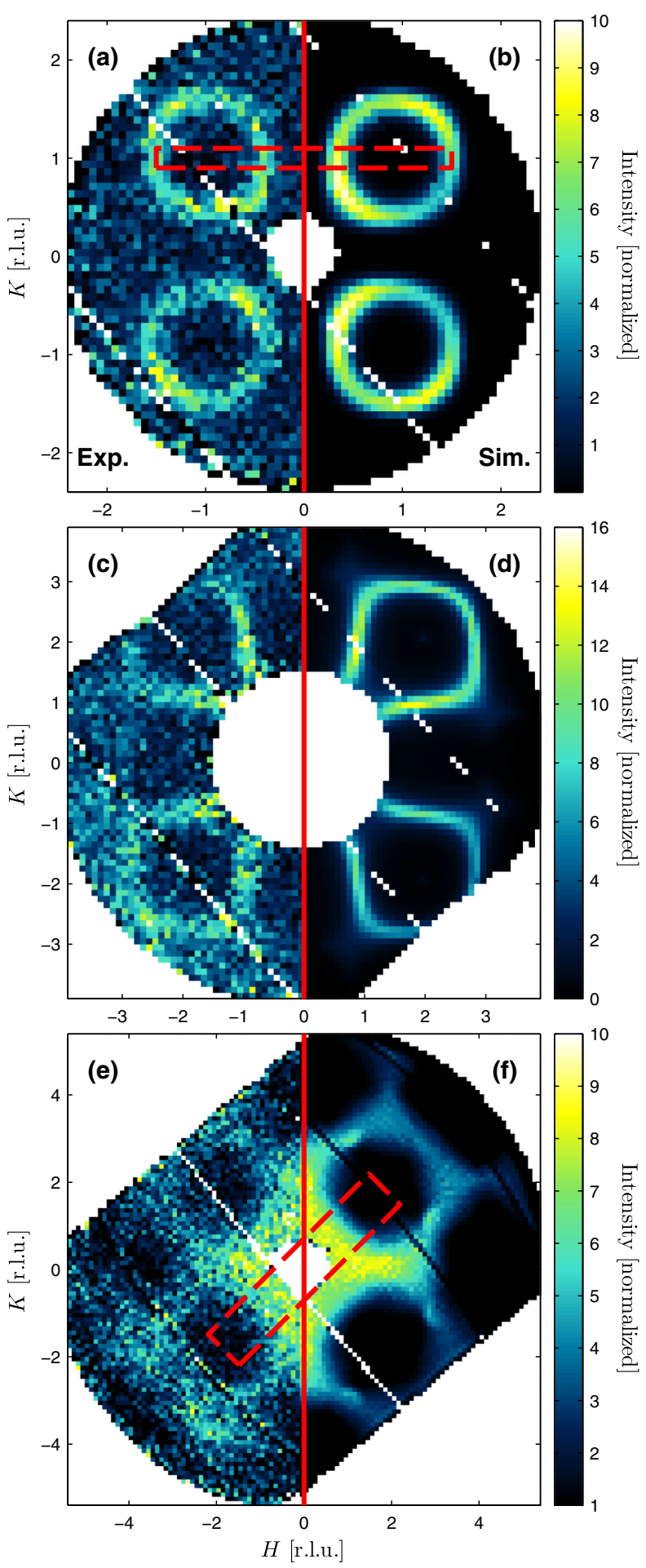

FIG. 3 (color online). Comparison of data (left half) and MCPHASE simulations (right half). (a) $E_{i}=25 \mathrm{meV}$, averaged with $L$ varying between 0.9 and 1.1 r.l.u. (c) $E_{i}=50 \mathrm{meV}$, averaged between 1.9-2.1 r.l.u. in $L$. (e) $E_{i}=80 \mathrm{meV}$, averaged between 31-35 meV in energy. A background has been subtracted for this data set only. Energy varies from $12 \mathrm{meV}$ at the center to $4 \mathrm{meV}$ at the edges in (a)-(b), 20 to $10 \mathrm{meV}$ in (c)-(d). $L$ varies from 4.5 in the center to 2 at the edges in (e)-(f). (b), (d), and (f) are simulations performed with $J=8.1$ and $D=0 \mathrm{meV}$. Dashed boxes represent regions of interest analyzed in cuts shown in Fig. 4. 


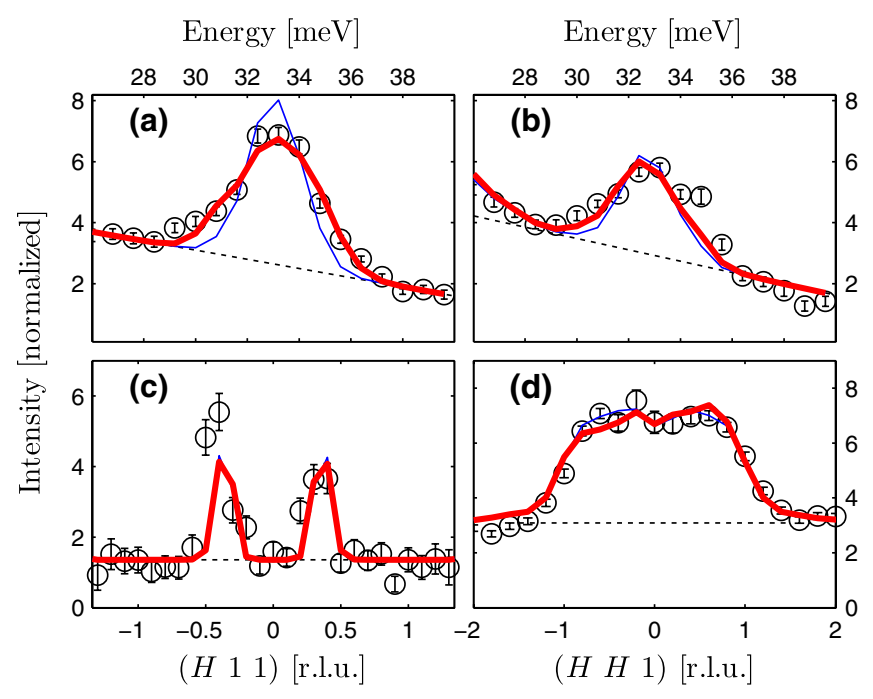

FIG. 4 (color online). Analysis of specific regions of $(\vec{Q}, \omega)$ to determine the best values of $J$ and $D$. Thick red lines represent the best fit of the MCPHASE simulations with $J=8.22(2)$ and $D=1.5(1) \mathrm{meV}$, thin blue lines, are $J=8.22(2)$ and $D=0 \mathrm{meV}$, dashed black lines are background. (a) and (b) Energy scans at $\sim 4 J$ obtained by averaging data over $1.5<$ $t(\vec{Q})<1.7$ and $0.7<t(\vec{Q})<0.9$, respectively [22]. (c) Wave vector dependence along $H$ averaged for $0.9 \leq K \leq 1.1$, $0.9 \leq L \leq 1.1$, and $6 \leq E \leq 8$ [box, Figs. 3(a)-3(b)]. (d) Wave vector dependence along $(H H 1)$ averaged for $-0.5 \leq(K \bar{K} 1) \leq$ $0.5,0 \leq L \leq 6$, and $28 \leq E \leq 35$ [box, Figs. 3(e)-3(f)].

is dominated by nearest-neighbor Heisenberg exchange. Simulations of $S(\vec{Q}, \omega)$ were also performed using the form factor for the putative orbital ordered state [14]: over the measured range of $(\vec{Q}, \omega)$ the results were essentially identical to those performed using the isotropic form factor.

However, it is apparent that the effects of the DMI are more pronounced in specific regions of the spin-wave dispersion, Fig. 1, and may not necessarily reveal themselves in cuts of the type shown in Fig. 3. We thus analyzed data from specific regions of the spin-wave dispersion, where the effects of the DMI were expected to be more significant. Figure 4 shows data extracted as cuts in both energy [(a) and (b)] and wave vector [(c) and (d)]. MCPHASE simulations were then fitted to this data to explore the dependence of the calculated scattering on $J$ and $D$. One complication in performing simulations for finite values of $D$ is that, as shown in Fig. 1(d), the spin-wave energy is dependent on the spin moment direction. As no external field was applied in the experiment, in the simulations we assumed domain coexistence and averaged the simulations over a set of easy-axis $\langle 100\rangle$-type domains.

For our data set, greatest sensitivity to the values of $J$ and $D$, with minimal contamination from phonon scattering, was obtained in energy cuts for energies $\sim 4 J$. The best fits of the MCPHASE simulations to energy scans around $\sim 4 J$, represented by the red lines in Fig. 4(a) and 4(b), were obtained with $J=8.22(2)$ and $D=1.5(1) \mathrm{meV}$, with $\chi^{2}=2.9$ and $\chi^{2}=6.7$ for (a) and (b), respectively. Constraining the value of $D=0$ produced significantly worse fits, as represented by the blue lines, with $\chi^{2}=14$ and $\chi^{2}=13$ for (a) and (b), respectively. The optimal values were found to be consistent with data analyzed from other regions of $(\vec{Q}, \omega)$, albeit with less sensitivity to $D$, as illustrated in Fig. 4(c) and 4(d).

The ratio of $D / J=0.18(1)$ determined in our study establishes the existence of a large finite $\mathrm{DMI}$ in $\mathrm{Lu}_{2} \mathrm{~V}_{2} \mathrm{O}_{7}$. Its value is roughly half the ratio of $\sim 1 / 3$ used in the analysis of the magnon Hall effect data [11]. It is clearly substantially larger than the value 0.004-0.005 obtained from other modeling of the thermal transport data [17] or the value 0.05 obtained from DFT calculations [16]. However, our determination of the leading term in the Hamiltonian, $J=8.22(2) \mathrm{meV}$, is in reasonable accord with the corresponding value of $J=7.1 \mathrm{meV}$ from DFT taking into account the accuracy of the computational method.

In conclusion, we have performed a neutron inelastic scattering experiment on the quantum ferromagnetic pyrochlore $\mathrm{Lu}_{2} \mathrm{~V}_{2} \mathrm{O}_{7}$. The well-defined spin-wave dispersion observed at all energies and wave vectors has allowed us to determine accurately for the first time the parameters of the Hamiltonian. Our data are to a remarkable extent accounted for by a minimal model of isotropic nearest-neighbor Heisenberg exchange with $J=8.22(2) \mathrm{meV}$ and with a DMI given by $D=$ 1.5(1) $\mathrm{meV}$. The existence of a large DMI term in $\mathrm{Lu}_{2} \mathrm{~V}_{2} \mathrm{O}_{7}$ may be taken as evidence in favor of the reported interpretation of the magnon Hall effect data [11]. Our value of $D / J$ is roughly half of the value required to explain the thermal transport data within the model used by Onose et al. [13] and is much larger than provided by other theoretical studies $[16,17]$. We hope that our results will stimulate further theoretical studies to explore this specific aspect, as well as wider questions such as the role of the topological band structure, of this intriguing material. Our data show that even in the quantum limit, well-defined spin waves can propagate on the highly frustrated pyrochlore lattice in the presence of nearest-neighbor ferromagnetic exchange. This situation contrasts starkly with the classical spin ices on the same lattice, where the long-range dipolar interaction gives rise to an effective ferromagnetic coupling and monopolar elementary excitations $[4,5]$.

The research was supported by the EPSRC and the Paul Scherrer Institute, and part of the research leading to these results has received funding from the European Community's Seventh Framework Programme (FP7/ 2007-2013) under Grant Agreement No. 312284. We thank Steve Bramwell for useful discussions, and acknowledge the help of Zhuo Feng, Simon Ward, and the ISIS technicians. 
[1] J. S. Gardner, M. J. P. Gingras, and J. E. Greedan, Rev. Mod. Phys. 82, 53 (2010).

[2] M. J. Harris, S. T. Bramwell, D. F. McMorrow, T. Zeiske, and K. W. Godfrey, Phys. Rev. Lett. 79, 2554 (1997).

[3] S. T. Bramwell and M. J. P. Gingras, Science 294, 1495 (2001).

[4] C. Castelnovo, R. Moessner, and S. L. Sondhi, Nature (London) 451, 42 (2008).

[5] S. T. Bramwell, S. R. Giblin, S. Calder, R. Aldus, D. Prabhakaran, and T. Fennell, Nature (London) 461, 956 (2009).

[6] A. B. Harris, A. J. Berlinsky, and C. Bruder, J. Appl. Phys. 69, 5200 (1991).

[7] M. Hermele, M. P. A. Fisher, and L. Balents, Phys. Rev. B 69, 064404 (2004).

[8] K. A. Ross, L. Savary, B. D. Gaulin, and L. Balents, Phys. Rev. X 1, 021002 (2011).

[9] O. Benton, O. Sikora, and N. Shannon, Phys. Rev. B 86, 075154 (2012).

[10] N. Nagaosa, J. Sinova, S. Onoda, A. H. McDonald, and N. P. Ong, Rev. Mod. Phys. 82, 1539 (2010).

[11] Y. Onose, T. Ideue, H. Katsura, Y. Shiomi, N. Nagaosa, and Y. Tokura, Science 329, 297 (2010).

[12] H. D. Zhou, E.S. Choi, J. A. Souza, J. Lu, Y. Xin, L. L. Lumata, B. S. Conner, L. Balicas, J. S. Brooks, J. J.
Neumeier, and C. R. Wiebe, Phys. Rev. B 77, 020411 (2008).

[13] T. Ideue, Y. Onose, H. Katsura, Y. Shiomi, S. Ishiwata, N. Nagaosa, and Y. Tokura, Phys. Rev. B 85, 134411 (2012).

[14] H. Ichikawa, L. Kano, M. Saitoh, S. Miyahara, N. Furukawa, J. Akimatsu, T. Yokoo, T. Matsumura, M. Takeda, and K. Hirota, J. Phys. Soc. Jpn. 74, 1020 (2005).

[15] L. Zhang, J. Ren, J.-S. Wang, and B. Li, Phys. Rev. B 87, 144101 (2013).

[16] H. J. Xiang, E. J. Kan, M.-H. Whangbo, C. Lee, Su-Huai Wei, and X. G. Gong, Phys. Rev. B 83, 174402 (2011).

[17] A. Mook, J. Henk, and I. Mertig, Phys. Rev. B 89, 134409 (2014).

[18] R. I. Bewley, R. S. Eccleston, K. A. McEwen, S. M. Hayden, M. T. Dove, S. M. Bennington, J . R. Treadgold, and R. L. S. Coleman, Physica (Amsterdam) 385B, 1029 (2006).

[19] Available at http://www.mantidproject.org.

[20] Available at http://horace.isis.rl.ac.uk.

[21] M. Rotter, S. Kramp, M. Loewenhaupt, E. Gratz, W. Schmidt, N. M. Pyka, B. Hennion, and R.v.d. Kamp, Appl. Phys. A 74, s751 (2002).

[22] See Supplemental Material at http://link.aps.org/ supplemental/10.1103/PhysRevLett.113.047202 for more information on the data treatment employed to produce the cuts of the data. 